\title{
ATUAÇÃO DA FISIOTERAPIA PÉLVICA NO TRATAMENTO DAS DISFUNÇÕES MICCIONAIS E SEXUAIS EM PACIENTES PROSTATECTOMIZADOS: REVISÃO INTEGRATIVA
}

\section{PELVIC PHYSIOTHERAPY PERFORMANCE IN THE TREATMENT OF VOIDING AND SEXUAL DYSFUNCTIONS IN PROSTATECTOMIZED PATIENTS: INTEGRATIVE REVIEW} \author{
Cavalcante $^{1}$ (D) Luandson José da Silva $^{2}$, , Rosimeire Gomes Nogueira ${ }^{3}$ (D) \\ ${ }^{1}$ Faculdade de Medicina Estácio de Juazeiro do Norte, Juazeiro do Norte, CE, Brasil. \\ ${ }^{2}$ Universidade Federal de Pernambuco, Recife, PE, Brasil. \\ ${ }^{3}$ Centro Universitário Fametro, Fortaleza, CE, Brasil. \\ *cidanogueira91@ hotmail.com
}

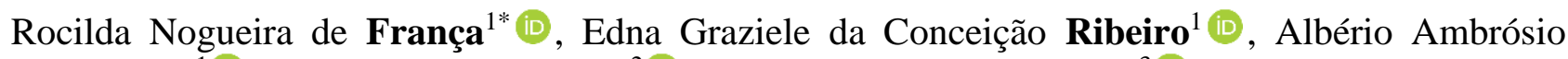

\section{RESUMO}

Nos últimos anos houve prevalência do câncer de próstata na população masculina, atingindo principalmente homens com mais de 65 anos. O principal tratamento cirúrgico é a prostatectomia radical total. Podendo ocorrer, como qualquer outra cirurgia, riscos e consequências danosas, pois o procedimento pode prejudicar o sistema urogenital. A fisioterapia pélvica atua como tratamento conservador, visando à reabilitação da função miccional e sexual desses indivíduos. Trata-se de uma revisão integrativa da literatura, com o objetivo de realizar o levantamento das intervenções fisioterapêuticas usadas nas disfunções causadas pela cirurgia, das publicações dos últimos 10 anos. A busca deu-se pelas bases de dados: BVS, Scielo, Pubmed e Pedro, através dos descritores, e suas combinações, Prostatectomia, Fisioterapia, Incontinência Urinária, e Disfunção erétil. Foram encontrados 337 artigos com o cruzamento dos descritores, porém apenas 184 foram publicados nos últimos 10 anos. A amostra final, após aplicabilidade dos critérios de inclusão e exclusão, resultou-se em 12 artigos. Observou-se o treinamento dos músculos do assoalho pélvico, a estimulação elétrica, a biofeedback, pilates e terapia comportamental como intervenções fisioterapêuticas utilizadas no tratamento conservador; percebeu-se que as terapias associadas apresentaram maior eficácia frente à disfunção miccional, porém na disfunção sexual a fisioterapia pélvica não tem efeito total sobre a disfunção erétil; podendo concluir-se que as intervenções fisioterapêuticas têm grande importância no tratamento das disfunções miccionais e sexuais, pois possibilita aos pacientes o retorno das suas funções urinárias, apesar de não promover melhora total da sua função sexual.

Pallavras-chave: Disfunção Erétil. Fisioterapia. Incontinência Urinária. Prostatectomia.

\section{ABSTRACT}

In recent years there has been a prevalence of prostate cancer in the male population, mainly affecting men over 65 years of age. The main surgical treatment is total radical prostatectomy. As with any other surgery, risks and harmful consequences may occur, as the procedure can harm the urogenital system. Pelvic physiotherapy acts as a conservative treatment, aimed at rehabilitating the voiding and sexual function of these individuals. This is an integrative literature review, with the aim of conducting a survey of physical therapy interventions used in disorders caused by surgery, published in the last 10 years. The search took place in the following databases: BVS, Scielo, Pubmed and Pedro, through the descriptors and their combinations, Prostatectomy, Physiotherapy, Urinary Incontinence, and Erectile Dysfunction. A total of 337 articles were found with the crossing of descriptors, but only 184 were published in the last 10 years. The final sample, after applicability of the inclusion and exclusion criteria, resulted in 12 articles. Pelvic floor muscle training, electrical stimulation, biofeedback, pilates and behavioral therapy were observed as physical therapy interventions used in conservative treatment; it was noticed that the associated therapies were more effective against voiding dysfunction, however, in sexual dysfunction, pelvic physiotherapy does not have a full effect on erectile dysfunction; it can be concluded that physiotherapeutic interventions are of great importance in the treatment of voiding and sexual dysfunctions, as it enables patients to return to their urinary functions, despite not promoting a total improvement in their sexual function.

Keywords: Erectile Dysfunction. Physiotherapy. Urinary incontinence. Prostatectomy. 


\section{INTRODUÇÃO}

A próstata é um órgão de composição fibromuscular e glandular, estando situada inferiormente à bexiga, normalmente pesa aproximadamente $20 \mathrm{~g}$, e contém a uretra posterior, medindo em torno de $2,5 \mathrm{~cm}$ de comprimento. Apresentando dois processos diferentes no seu crescimento, a hiperplasia, geralmente o mais comum, com caráter benigno e que se apresenta após 40 anos, e o câncer de próstata $(\mathrm{CaP})$, que afeta homens no início da terceira idade (TANAGHO; MCANICH, 2006; ZAIDAN; MULLER; SILVA, 2016).

Segundo o Instituto Nacional do Câncer, estima-se 65.840 novos casos de CaP a cada ano (2020 e 2022), sendo que homens acima de 55 anos com quadro de obesidades apresentam maior predisposição à doença. Apesar de esses dados serem alarmantes, esse aumento se dá pelo diagnóstico precoce, que aumenta a expectativa de vida (INCA, 2020).

De acordo com Santos et al. (2016), existem diversos tipos de tratamentos para o CaP, entre eles podemos destacar a prostatectomia radical (PR), que vem apresentando maior eficácia no tratamento desse tipo de câncer; a resolutividade do tumor vai depender de diversos fatores, dentre eles estão: estágio tumoral, nível de distinção do tumor, técnica cirúrgica e competência do cirurgião.

Assim como toda modalidade terapêutica, a PR possui efeitos e riscos significativos, ocasionando estenose uretral, incontinência urinária (IU) e disfunção erétil (DE), apresentando maior predominância, no pós-cirúrgico, a IU e a DE (GOULART; MIRANZI; GOULART, 2014).

A International Continence Society conceitua incontinência urinária como qualquer perda involuntária de urina. A IU ocorre devido à localização anatômica da próstata, no entanto após sua remoção o controle urinário será mantido pelo colo da bexiga e esfíncter urinário externo; devido às lesões, a junção uretrovesical será menos eficiente, tornando difícil o controle urinário, causando maior pressão sobre o esfíncter urinário externo, que por sua vez dependerá do bom funcionamento de suas fibras musculares estriadas. Além da lesão do esfíncter e da sua inervação, os músculos do assoalho pélvico também serão afetados (ZAIDAN; SILVA, 2014; SANTOS et al., 2016).

De acordo Prota et al. (2010) a DE após a PR é causada por lesões no nervo cavernoso, quando isso ocorre os músculos lisos e tecidos vizinhos sofreram hipóxia e fibrose, além do comprometimento vascular naquela região, a fraqueza da musculatura do assoalho também pode estar envolvida.

As disfunções causadas pela cirurgia afetam diretamente a qualidade de vida (QV) do paciente, gerando um impacto negativo no seu bem-estar físico, social e psicológico, além de complicações como infecção no trato urinário, dermatites, constrangimento, causando profunda alteração na sua autoestima (SCARPELINI et al., 2014).

Pacientes submetidos à PR necessitam precocemente de reabilitação; apesar de existir diversos tipos de tratamento, a área que vem tendo maior destaque, atualmente, é a fisioterapia pélvica, que por meio de intervenções fisioterápicas, visam atender às necessidades desses indivíduos, trazendo benefícios à sua vida social e afetiva.

Frente à prevalência do $\mathrm{CaP}$ na população masculina e devido o tratamento cirúrgico causar disfunções miccionais e sexuais, faz-se necessário estudos que destacam a importância do tratamento conservador após a PR.

Dessa forma, o objetivo do presente estudo é realizar levantamento sobre quais intervenções fisioterápicas são utilizadas nas disfunções miccionais e sexuais, bem como destacar e discutir os benefícios trazidos por elas. Além do mais, esse estudo poderá despertar o profissional para realizar mais pesquisas neste contexto, pois a reabilitação fisioterapêutica é de suma importância aos portadores de sequelas após PR. 


\title{
METODOLOGIA
}

\section{Tipo de estudo}

Trata-se de uma revisão integrativa da literatura, com abordagem em pesquisa de artigos sobre atuação da fisioterapia pélvica no tratamento das disfunções miccionais e sexuais em pacientes prostatectomizados. De acordo com Souza, Silva e Carvalho (2010) será necessário seguir seis etapas distintas para a construção desta revisão integrativa: 1) Elaboração da pergunta norteadora; 2) busca ou amostragem na literatura; 3) coleta de dados; 4) análise crítica dos estudos incluídos; 5) discussão dos resultados; 6) Apresentação da revisão integrativa:

\begin{abstract}
"A revisão integrativa da literatura consiste na construção de uma análise ampla da literatura, contribuindo para discussões sobre métodos e resultados de pesquisas, assim como reflexões sobre a realização de futuros estudos. O propósito inicial deste método de pesquisa é obter um profundo entendimento de um determinado fenômeno baseando-se em estudos anteriores (MENDES; SILVEIRA; GALVÃO, 2008, p. 4)".
\end{abstract}

\section{Critérios de elegibilidade e seleção dos artigos}

Após a escolha do tema em questão, realizou-se uma busca inicial nas seguintes bases de dados: Biblioteca virtual da saúde (BVS), Pubmed, Scientific Eletronic Library Online (Scielo) e PEDRO, sendo formuladas palavras chaves de acordo com a qualificação do Descritores em Ciências da Saúde (DESC), utilizando os descritores: Prostatectomy, Physical Therapy, Urinary Incontinence and Erectile Dysfunction. Posteriormente, para as combinações das palavras, utilizou-se do operador booleano AND.

Estabeleceu-se como critérios de inclusão: estudos publicados em periódicos da língua inglesa, portuguesa e espanhola, entre os anos de 2008 e 2018, estudos de intervenção (estudos randomizados ou não, estudos clínicos, estudos de casos), estudos que abordassem apenas a PR como intervenção cirúrgica no tratamento do $\mathrm{CaP}$ e tratamento conservador apenas no pós-operatório.

Dessa forma, os estudos de revisão sistemática ou literária, estudos envolvendo intervenções conservadoras no pré-operatório ou em outro tipo de procedimento cirúrgico foram estabelecidos como critérios de exclusão.

A coleta de dados foi realizada seguindo o fluxograma a seguir, de elaboração do próprio autor.

Figura 1 - Fluxograma representando o processo e as etapas de seleção dos artigos incluídos na revisão

\begin{tabular}{|l|l|l|}
\hline \multicolumn{1}{|l|}{$\begin{array}{l}\text { Buscas nas bases de dados eletrônicas: } \\
\text { BVS, PUBMED, SciELO, PEDRO. }\end{array}$} & $\begin{array}{l}\text { Descritores: Prostatectomy, Physical, } \\
\text { Therapy, Urinary Incontinence and } \\
\text { Erectile Dysfunction. }\end{array}$ \\
\cline { 1 - 2 } $\begin{array}{l}\text { Inclusão: Estudos de intervenção, estudos } \\
\text { randomizados ou não, estudos clínicos, } \\
\text { estudo de casos publicados entre os anos } \\
\text { de } 2008 \text { e } 2018 .\end{array}$ & $\begin{array}{l}\text { Exclusão: Revisão sistemática e } \\
\text { literária, estudos de intervenção no } \\
\text { pré-operatório da PR ou envolvendo } \\
\text { outros tratamentos cirúrgicos. }\end{array}$ \\
\hline
\end{tabular}

Fonte: os autores. 


\section{Fases da coleta}

A pesquisa ocorreu no período entre agosto de 2018 e fevereiro de 2019, frente às escolhas dos critérios de seleção dos artigos (bases de dados, ano de publicação e descritores), sendo feito uma visita nos respectivos locais, realizando-se assim o processo de busca de artigos sobre a fisioterapia no tratamento conservador após a PR. A seleção dos artigos foi feita, inicialmente, pela leitura superficial do título e resumo, posteriormente, realizou-se a leitura aprofundada e selecionados os dados para compor a pesquisa.

Os achados foram agrupados em categorias temáticas e, posteriormente, abordados de forma descritiva e exploratória, possibilitando responder à questão norteadora do estudo.

\section{Análises dos dados}

Os resultados encontrados foram expostos em forma de quadros, utilizando os subtítulos para análise dos dados: Título do estudo, autor/ano, tipo de estudo, objetivos e resultados.

\section{Aspectos éticos e legais da pesquisa}

Foram obedecidos os aspectos éticos da pesquisa, dispostos na Resolução 510/16 do Conselho Nacional de Saúde, no que se refere à fidedignidade dos resultados apresentados pelos autores estudados; por tratar-se de uma revisão de literatura e ter utilizado o domínio público, não houve necessidade de ser submetido ao comitê de ética.

\section{RESULTADOS E DISCUSSÃO}

Com o cruzamento dos descritores, foram encontradas 337 publicações; após a filtragem do ano das publicações, 2008 a 2018, os idiomas escolhidos como critério de inclusão, encontram-se 184 artigos, sendo 38 da BVS, 05 da Scielo, 07 Pedro, 134 Pubmed. No entanto, 58 artigos foram excluídos, pois se tratavam de revisões, 09 por estarem duplicados e 99 artigos onde os títulos e resumos não estavam de acordo com a questão proposta. Resultando-se, dessa pré-seleção, resultou na avaliação de 18 textos.

Considerando os critérios de inclusão e exclusão, excluíram-se 06 artigos e selecionou-se apenas 12 estudos para análise e discussão.

O fluxograma seguinte (Figura 2) demonstra a estratégia de busca e a seleção dos artigos que foram agregados nesta revisão de literatura.

Figura 2 - Fluxograma representando o processo de seleção dos artigos

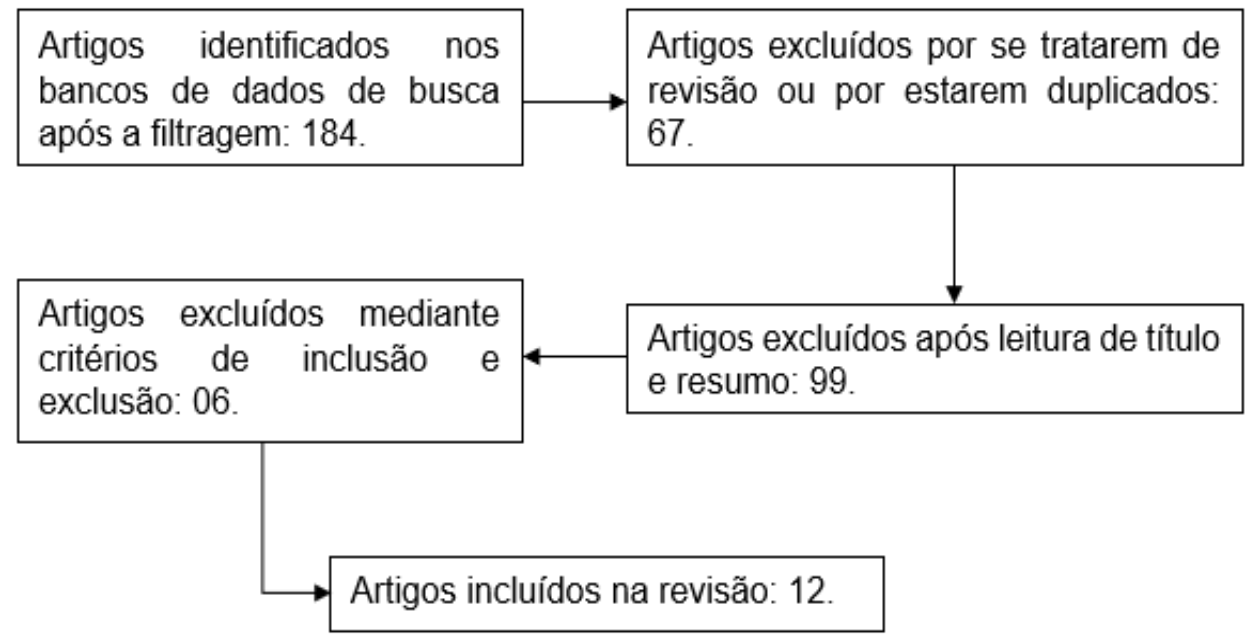

Fonte: os autores. 
A seguir, nos quadros, há a exposição do resumo dos artigos selecionados para este estudo de revisão de literatura, contendo o título, autor/ano, tipo de estudo, objetivos e resultados; no qual auxiliarão na leitura da discussão e resultado do estudo.

Quadro 1 - Refere-se aos estudos com intervenções fisioterapêuticas associadas no tratamento da IU

\begin{tabular}{|c|c|c|c|c|}
\hline Título do Estudo & Autor / Ano & $\begin{array}{l}\text { Tipo de } \\
\text { Estudo }\end{array}$ & Objetivos & Resultados \\
\hline $\begin{array}{l}\text { Terapia } \\
\text { comportamental com } \\
\text { ou sem biofeedback e } \\
\text { estimulação elétrica } \\
\text { do assoalho pélvico } \\
\text { para incontinência } \\
\text { pós-prostatectomia } \\
\text { persistente: um estudo } \\
\text { controlado } \\
\text { randomizado }\end{array}$ & $\begin{array}{l}\text { Goode et al. } \\
(2011)\end{array}$ & Randomizado & $\begin{array}{l}\text { Avaliar a eficácia da terapia } \\
\text { comportamental para reduzir } \\
\text { a IU pós-PR persistente e } \\
\text { determinar se as tecnologias } \\
\text { de biofeedback (BFB) e } \\
\text { estimulação elétrica (EE) do } \\
\text { assoalho pélvico aumentam a } \\
\text { eficácia da terapia } \\
\text { comportamental }\end{array}$ & $\begin{array}{l}\text { Menos episódios de IU. } \\
\text { A adição de BFB e EE do } \\
\text { assoalho pélvico não } \\
\text { resultou em maior } \\
\text { eficácia }\end{array}$ \\
\hline $\begin{array}{lr}\text { Efeito da estimulação } \\
\text { elétrica do assoalho } \\
\text { pélvico e r do } \\
\text { biofeedback } & \text { na } \\
\text { recuperação } & \text { da } \\
\text { continência urinária } \\
\text { após prostatectomia } \\
\text { radical }\end{array}$ & $\begin{array}{l}\text { Ahmed; } \\
\text { Mohammed; } \\
\text { Amansour, } \\
(2012)\end{array}$ & $\begin{array}{l}\text { Prospectivo, } \\
\text { randomizado e } \\
\text { controlado }\end{array}$ & $\begin{array}{l}\text { Avaliar o efeito de exercícios } \\
\text { da musculatura pélvica } \\
(\text { EMP), EE e BFB em IU após } \\
\text { PR }\end{array}$ & $\begin{array}{l}\text { EE e BFB têm um efeito } \\
\text { positivo significativo na } \\
\text { duração e no grau de IU e } \\
\text { QV }\end{array}$ \\
\hline $\begin{array}{lr}\text { Avaliação } & \text { de } \\
\text { estratégias } & \text { de } \\
\text { fisioterapia } & \text { para } \\
\text { recuperação } & \text { da } \\
\text { continuidade } & \text { urinária } \\
\text { após prostatectomia }\end{array}$ & $\begin{array}{l}\text { Santos et al. } \\
(2017)\end{array}$ & $\begin{array}{l}\text { Estudo de } \\
\text { intervenção, } \\
\text { prospectivo e } \\
\text { randomizado }\end{array}$ & $\begin{array}{l}\text { Analisar a aplicação de } \\
\text { técnicas de fisioterapia na } \\
\text { recuperação de incontinência } \\
\text { urinária após a PR }\end{array}$ & $\begin{array}{l}\text { Ambas as intervenções } \\
\text { proporcionaram melhora } \\
\text { no grau de IU dentro de } 2 \\
\text { meses }\end{array}$ \\
\hline
\end{tabular}

Fonte: os autores.

Em um estudo feito por Goode et al. (2011), foi aplicada a terapia comportamental em 208 pacientes, que consiste na mudança de hábitos de vida desses pacientes, permitindo à reeducação do controle cortical sobre bexiga, tornando perceptível o momento correto do ato de urinar; a técnica foi avaliada isoladamente ou associada com eletroestimulação (EE) e biofeedback (BFB).

Estes autores apontam que a terapia comportamental mostrou-se eficaz no tratamento da IU, além de proporcionar uma melhora da QV desses indivíduos, no entanto, a associação do BFB e EE não provocaram efeitos maiores sobre os resultados obtidos apenas com a terapia comportamental. Em discordância, Carvalho, Silva e Silveira (2018) ressaltam a importância de técnicas associadas à terapia comportamental, principalmente àqueles pacientes que possuem dificuldade para iniciar a contração dos músculos do assoalho pélvico (MAP).

Ahmed, Mohammed e Amansour (2012) avaliaram o uso do BFB com a EE em 80 pacientes, sendo divididos em três grupos. $\mathrm{O}$ grupo que recebeu as técnicas associadas obteve ligeiramente aumento da força da musculatura pélvica e, com isso, atingiu a continência urinária mais rápida, isso ocorre devido ao estímulo provocado pelas duas intervenções, pois quando o músculo aprende a trabalhar de forma mais eficiente, torna-se mais fácil adquirir um controle pélvico autonômico. Em concordância, Mariotti et al. (2015) utilizaram as mesmas técnicas em 120 pacientes e obtiveram resultados semelhantes.

Santos et al. (2017) realizaram um estudo com 13 pacientes em uma clínica de fisioterapia pélvica em Fortaleza-CE, analisando no grupo de tratamento o uso BFB associado aos exercícios da musculatura pélvica (EMP) e o grupo de controle recebeu apenas EMP, concluíram que a associação de técnicas fisioterapêuticas podem ter desfechos mais positivos em comparação com exercícios 
isolados; porém Kannan et al. (2019b) relataram que os efeitos do EMP com BFB são incertos, mesmo com resultados promissores ao final do tratamento, devido às poucas evidências sobre as técnicas.

Apesar de o primeiro estudo apresentar resultados diferentes em relação à combinação de técnicas fisioterápicas, mesmo com uma amostra consideravelmente satisfatória, nota-se que a associação dessas técnicas pode potencializar o tratamento desses indivíduos, promovendo a continência mais rápida, uma vez que os pacientes, ao ver ligeiramente resultados com o tratamento, adquirem ainda mais confiança e se empolgam em colaborar com as instruções e os deveres domiciliares, desse modo, o tratamento é mais satisfatório, porque possibilita o retorno mais rápido às suas atividades diárias, além de contribuir para uma maior positividade na QV.

Quadro 2 - Refere-se aos estudos com intervenções fisioterápicas isoladas no tratamento de IU pós PR

\begin{tabular}{|c|c|c|c|c|}
\hline Título do Estudo & $\begin{array}{c}\text { Autor / } \\
\text { Ano }\end{array}$ & $\begin{array}{l}\text { Tipo de } \\
\text { Estudo }\end{array}$ & Objetivos & Resultados \\
\hline $\begin{array}{l}\text { Efeito em longo prazo do } \\
\text { biofeedback pós-operatório } \\
\text { do assoalho pélvico na } \\
\text { continência em homens } \\
\text { submetidos à prostatectomia } \\
\text { radical: um estudo } \\
\text { prospectivo randomizado e } \\
\text { controlado }\end{array}$ & $\begin{array}{l}\text { Ribeiro et } \\
\text { al. }(2010)\end{array}$ & $\begin{array}{l}\text { Prospectivo, } \\
\text { randomizado e } \\
\text { controlado. }\end{array}$ & $\begin{array}{l}\text { Testar a eficácia do } \\
\text { treinamento do músculo } \\
\text { do assoalho pélvico } \\
\text { (TMAP), BFB - } \\
\text { pélvico para melhorar a } \\
\text { incontinência urinária } \\
\text { nos } 12 \text { meses após a PR }\end{array}$ & $\begin{array}{l}\text { Mudanças significativas } \\
\text { em ambos os grupos em } \\
\text { termos de sintomas do } \\
\text { trato urinário inferior, e } \\
\text { força dos MAP }\end{array}$ \\
\hline $\begin{array}{l}\text { O treinamento dos músculos } \\
\text { do assoalho pélvico guiado } \\
\text { por fisioterapeuta aumenta a } \\
\text { qualidade de vida dos } \\
\text { pacientes após a } \\
\text { prostastectomia radical? Um } \\
\text { estudo clínico randomizado }\end{array}$ & $\begin{array}{l}\text { Nilssen et } \\
\text { al. (2012) }\end{array}$ & Randomizado & $\begin{array}{lrr}\text { Estudar o efeito do } & \text { do } \\
\text { TMAP guiado por } \\
\text { fisioterapeuta no pós- } \\
\text { operatório } & & \text { nos } \\
\text { parâmetros } & & \text { de } \\
\text { qualidade de vida } \\
\text { relacionada à saúde } \\
\text { (QVRS) em pacientes } \\
\text { tratados com PR }\end{array}$ & $\begin{array}{l}\text { Melhora a IU, porém isso } \\
\text { não refletiu em melhores } \\
\text { resultados nos } \\
\text { parâmetros de QVRS }\end{array}$ \\
\hline $\begin{array}{l}\text { Eletroestimulação } \\
\text { incontinência urinária pós- } \\
\text { prostatectomia radical }\end{array}$ & $\begin{array}{l}\text { Santos et al. } \\
(2016)\end{array}$ & $\begin{array}{l}\text { Não } \\
\text { randomizado }\end{array}$ & $\begin{array}{l}\text { Avaliar os efeitos da EE } \\
\text { funcional endo-anal na } \\
\text { recuperação funcional } \\
\text { da continência urinária } \\
\text { de homens submetidos } \\
\text { à PR }\end{array}$ & $\begin{array}{l}\text { Redução da perda } \\
\text { urinária; a pressão dos } \\
\text { MAP aumentou, a QV } \\
\text { melhorou nos aspectos às } \\
\text { limitações de atividades } \\
\text { diárias e relações } \\
\text { pessoais }\end{array}$ \\
\hline $\begin{array}{l}\text { Os efeitos do método pilates } \\
\text { na força muscular do assoalho } \\
\text { pélvico com pacientes com } \\
\text { incontinência urinária pós- } \\
\text { postatectomia: um ensaio } \\
\text { clínico randomizado }\end{array}$ & $\begin{array}{l}\text { Gomes et } \\
\text { al. (2018) }\end{array}$ & $\begin{array}{l}\text { Ensaio clínico } \\
\text { randomizado. }\end{array}$ & $\begin{array}{l}\text { Avaliar os efeitos de um } \\
\text { exercício de programas } \\
\text { de pilates comparado ao } \\
\text { protocolo convencional } \\
\text { de TMAP na força } \\
\text { muscular do assoalho } \\
\text { pélvico (FMAP) em } \\
\text { pacientes com IU pós- } \\
\text { prostatectomia }\end{array}$ & $\begin{array}{lr}\text { O método } & \text { pilates } \\
\text { promoveu } & \text { resultados } \\
\text { semelhantes na proporção } & \\
\text { de pacientes } & \text { totalmente } \\
\text { continentes } & \text { quando } \\
\text { comparado ao } & \text { TMAP } \\
\text { convencional } 4 \text { meses PR }\end{array}$ \\
\hline
\end{tabular}

Fonte: os autores.

Um estudo randomizado realizado por Ribeiro et al. (2010) mostra que, 36 pacientes receberam tratamento para testar a eficácia do BFB, já o grupo controle contou com 37 doentes realizando apenas exercícios domiciliares. $\mathrm{O}$ tratamento com o BFB proporciona melhoria quanto à gravidade da IU, desaparecimento dos sintomas miccionais e aumento da força muscular. Já em relação à QV não foi possível observar resultados satisfatórios. Em concordância, um estudo mais recente realizado por Kim et al. (2019), foi utilizado o BFB extracorpóreo e, ao final do tratamento, as repercussões dos resultados foram idênticas. 
Em concordância com os autores acima, Nilssen et al. (2012) também relataram em seu estudo, resultado negativo em relação à QV dos pacientes, apesar de seu estudo utilizar uma intervenção diferente, onde foi aplicado o treinamento da musculatura pélvica (TMAP); sendo possível observar que as técnicas isoladas estudadas pelos autores citados, possuem déficits beneficiários sobre a QV desses doentes ou talvez esses indivíduos tentem mascarar o impacto da IU na $\mathrm{QV}$, devido à ansiedade causada pela resolução do Cap ou por darem maior importância a DE.

Destarte, os autores acima concluíram que o TMAP foi considerado impactante no tratamento da IU, o que também se pode observar em estudo realizado por Sayilan e Ozbas (2018), onde constataram que ao iniciar o tratamento no pré-operatório esses indivíduos também serão beneficiados quanto à QV.

Contrapondo os autores acima, Ribeiro et al. (2010), Nilssen et al. (2012) e Gomes et al. (2018) avaliaram os efeitos do pilates sobre a IU, comparando os resultados com o tratamento convencional do TMAP; o estudo envolveu 104 pacientes. Ao final do tratamento, observou-se uma melhora significativa da QV em todos os grupos, independente da técnica utilizada.

Além dos benefícios da melhora da QV já citados, todos os pacientes tornaram-se continentes, dessa forma, conclui-se que, tanto o pilates quanto o TMAP são eficazes no tratamento das disfunções miccionais desses doentes. A mesma hipótese já tinha sido avaliada por Pedriali et al. (2015), porém o estudo envolveu uma amostra menor de pacientes.

Possivelmente, o efeito do pilates sobre a IU, foi devido o aumento da força muscular e resistência, mediante à contração sinérgica do MAP, com transverso abdominal, adutores do quadril, glúteos e diafragma, sendo recrutados simultaneamente.

Santos et al. (2016) avaliaram o efeito da EE sobre a IU em uma amostra de 03 doentes prostatectomizados, utilizando uma corrente bipolar simétrica por meio do aparelho dualpex, por 10 sessões; todas as sessões tiveram um tempo de duração de 20 minutos, com os seguintes parâmetros iniciais: Frequência: 10hz, Largura de pulso: 250, sendo aumentados de acordo com o feedback do paciente ou visualização da contração muscular. Já em outro estudo feito por Zaidan e Silva (2016), no intuito de avaliar a mesma hipótese, porém com uma amostra de 16 pacientes e com período de atendimento maior, onde se utilizou uma corrente bifásica com diferentes parâmetros iniciais.

De acordo com os estudos pode-se concluir que a EE tem eficácia frente ao quadro da IU, devido à reeducação do controle dos esfíncteres e do períneo e por estimular o recrutamento das fibras rápidas e lentas, possibilitando, desta forma, um maior fortalecimento da musculatura pélvica. Entretanto, Kannan et al. (2019a) relatam que mesmo tendo eficácia no tratamento da IU, o uso clínico da EE pode ser limitado, devido à escassez de estudos que analisam o risco de disseminação das células cancerígenas.

Sendo assim, independente da intervenção fisioterapêutica utilizada, esses pacientes serão beneficiados quanto à resolução da IU, porém os benefícios em relação à QV só foram apresentados no tratamento utilizando Pilates e EE, nas demais técnicas avaliadas, os benefícios em relação à QV é algo ainda não comprovado.

Através de um estudo feito por Sighinolfi et al. (2009) avaliou-se o efeito de um programa de reabilitação pélvica sobre a IU, DE e climatúria, onde o programa envolveu várias técnicas fisioterapêuticas, dentre elas, BFB, EE, TMAP e mudanças comportamentais.

Os exercícios pélvicos vão melhorar a IU, mediante o controle do assoalho pélvico, que por sua vez, possibilitará o fechamento uretral primário, tornando possível à continência urinária; já na DE vão permitir uma melhora na ereção peniana frente ao favorecimento de um maior aporte sanguíneo para a região, sendo que a rigidez peniana também será facilitada, devido uma maior contração dos músculos isquiocavernosos, bulbocavernosos e intracavernoso.

A associação de técnicas utilizadas pelos autores enriqueceu ainda mais os efeitos citados acima, contribuindo para uma melhora significativa da IU, da DE e climatúria.

Prota et al. (2010) avaliaram os efeitos do BFB em 52 pacientes submetidos à $\mathrm{PR}$, tendo como conclusão efeitos positivos sobre à função erétil (FE) e IU; porém Perez et al. (2018) compararam os efeitos do BFB quando utilizado no pré e pós-operatório, mostrando que, apenas uma parte dos 
indivíduos submetidos à cirurgia, apresentaram melhora no seu quadro, àqueles pacientes que iniciaram o tratamento no pré-operatório preveniram maiores sequelas, pois a hipertrofia dos MAP auxiliou no procedimento cirúrgico contribuindo para menores lesões após a retirada da próstata.

Os autores acima interrogam a questão da IU está associada à DE, mas devido às poucas publicações comprovando essa hipótese, tal fato ainda não pode ser comprovado. Todavia, em outro estudo, os autores Laurienzo et al. (2018) também relataram a possibilidade da IU está associada a DE, pois esta pode interferir na realização sexual e provocar insatisfação sexual nesses indivíduos.

Quadro 3 - Refere-se aos estudos de intervenções utilizadas no tratamento das disfunções miccionais e sexuais

\begin{tabular}{|c|c|c|c|c|}
\hline Título do Estudo & $\begin{array}{c}\text { Autor / } \\
\text { Ano } \\
\end{array}$ & $\begin{array}{l}\text { Tipo de } \\
\text { Estudo } \\
\end{array}$ & Objetivos & Resultados \\
\hline $\begin{array}{lr}\text { Eficácia potencial } & \text { do } \\
\text { tratamento } & \text { de } \\
\text { reabilitação do assoalho } \\
\text { pélvicor } & \text { para } \\
\text { incontinência } & \text { pós- } \\
\text { prostatectomia pós- } & \text { pós- } \\
\text { operatória e disfunção } \\
\text { erétil }\end{array}$ & $\begin{array}{l}\text { Sighinolfi et } \\
\text { al. (2009) }\end{array}$ & Estudo de caso & $\begin{array}{l}\text { Avaliar o efeito de um } \\
\text { programa pélvico completo } \\
\text { de reabilitação do assoalho } \\
\text { pélvico em IU e DE, } \\
\text { incluindo climatúria }\end{array}$ & $\begin{array}{l}\text { Continência urinária e } \\
\text { uma melhora na FE } \\
\text { (função erétil) }\end{array}$ \\
\hline $\begin{array}{l}\text { Biofeedback pós- } \\
\text { operatório precoce do } \\
\text { assoalho pélvico } \\
\text { melhora a função erétil } \\
\text { em homens submetidos } \\
\text { à prostatectomia } \\
\text { radical: um estudo } \\
\text { prospectivo, } \\
\text { randomizado } \\
\text { controlado }\end{array}$ & $\begin{array}{l}\text { Prota et al. } \\
(2012)\end{array}$ & $\begin{array}{l}\text { Prospectivo, } \\
\text { randomizado e } \\
\text { controlado }\end{array}$ & $\begin{array}{l}\text { Avaliar o efeito do BFB na } \\
\text { recuperação da potência } \\
\text { sexual }\end{array}$ & $\begin{array}{l}\text { O estado de continência } \\
\text { urinária ajudou na } \\
\text { recuperação da FE; } \\
\text { pacientes continentes } \\
\text { apresentam uma maior } \\
\text { chance de serem potentes }\end{array}$ \\
\hline $\begin{array}{l}\text { Treinamento do } \\
\text { músculo do assoalho } \\
\text { pélvico para disfunção } \\
\text { erétil e climatúria } 1 \text { ano } \\
\text { após prostatectomia } \\
\text { radical poupadora de } \\
\text { nervo: um estudo } \\
\text { randomizado } \\
\text { controlado }\end{array}$ & $\begin{array}{l}\text { Geraerts et } \\
\text { al. }(2016)\end{array}$ & $\begin{array}{l}\text { Randomizado e } \\
\text { controlado }\end{array}$ & $\begin{array}{l}\text { Determinar se os pacientes } \\
\text { com DE persistente, mínimo } \\
\text { de } 12 \text { meses, } \\
\text { experimentaram uma } \\
\text { melhor recuperação da FE } \\
\text { com TMAP }\end{array}$ & $\begin{array}{l}\text { Obtiveram uma melhor } \\
\text { recuperação da FE. Além } \\
\text { disso, o TMAP teve um } \\
\text { efeito benéfico sobre a } \\
\text { climatúria }\end{array}$ \\
\hline $\begin{array}{l}\text { Viabilidade } \\
\text { aceitabilidade do } \\
\text { aconselhamento de } \\
\text { casal e treinamento } \\
\text { muscular do assoalho } \\
\text { pélvico após a operação } \\
\text { para câncer de próstata }\end{array}$ & $\begin{array}{l}\text { Karlsen et } \\
\text { al. }(2017)\end{array}$ & $\begin{array}{l}\text { Estudo de } \\
\text { intervenção }\end{array}$ & $\begin{array}{l}\text { Examinar a viabilidade e } \\
\text { aceitação da intervenção } \\
\text { Procan (Prostate Cancer), } \\
\text { que consiste em especialista } \\
\text { de aconselhamento sexual } \\
\text { para casais e TMAP } \\
\text { especialista }\end{array}$ & $\begin{array}{l}\text { A DE melhorou do início } \\
\text { ao moderado aos } 8 \text { meses } \\
\text { de seguimento e houve } \\
\text { melhora } \\
\text { funcionamento } \\
\text { médio, mas doxual } \\
\text { ligeiramente }\end{array}$ \\
\hline $\begin{array}{l}\text { Treinamento muscular } \\
\text { do assoalho pélvico e } \\
\text { estimulação elétrica } \\
\text { como reabilitação após } \\
\text { prostatectomia radical: } \\
\text { um estudo randomizado } \\
\text { controlado }\end{array}$ & $\begin{array}{l}\text { Laurienzo et } \\
\text { al. }(2018)\end{array}$ & $\begin{array}{l}\text { Randomizado } \\
\text { controlado }\end{array}$ & $\begin{array}{l}\text { Investigar o efeito de EE e a } \\
\text { formação da musculatura do } \\
\text { pavimento pélvico na força } \\
\text { muscular, IU e FE em } \\
\text { homens com cancro de } \\
\text { próstata tratados por PR }\end{array}$ & $\begin{array}{l}\text { Recuperação da força } \\
\text { muscular em todas as } \\
\text { intervenções. TMAP ou } \\
\text { EE não tiveram impacto } \\
\text { na recuperação da } \\
\text { continência urinária e da } \\
\text { FE }\end{array}$ \\
\hline
\end{tabular}

Fonte: os autores.

Em seu estudo, os autores Gerarts et al. (2016), avaliaram a eficácia do TMAP em 33 pacientes com DE e climatúria; o tratamento teve uma durabilidade de 3 meses e envolveu o TMAP associado à EE e orientações sobre exercícios domiciliares. Após a terapia os pacientes apresentaram aumento 
da força e contração muscular mais eficiente, consequentemente, alcançando maior tempo de ereção e evoluindo para uma menor gravidade de DE, obtendo também resolução da climatúria.

Laurienzo et al. (2018), em discordância aos autores acima, apresentaram resultados diferentes utilizando a mesma intervenção, onde foram incluídos, no estudo, 123 pacientes, durante 6 meses de tratamento. Os autores concluíram que, mesmo com o aumento da força muscular da musculatura pélvica ao final do tratamento, isso não repercute na melhora significativa da DE e IU, sendo possível observar efeitos maiores sobre a IU.

Em contribuição com os autores, Kannan et al. (2019b) também apresentaram resultados inconclusivos frente à recuperação da EE, quando submetidos à reabilitação com TMAP; ressaltando que o uso EE não é seguro e, portanto, deve ser utilizada apenas em pacientes terminais. Em divergência, Bispo, Nascimento e Soler (2019) afirmam que a EE é segura e eficaz no tratamento da disfunção sexual desses pacientes.

Denota-se que não há consenso sobre os benefícios trazidos pelo TMAP na DE, supostamente pelo impacto negativo gerado tanto pelas sequelas como também pelos fatores psicológicos, pois a satisfação sexual vai além do bem-estar físico; talvez esses indivíduos já apresentassem a DE devido à predisposição da idade.

Destarte, os autores Karlsen et al. (2017), observaram em 7 pacientes os efeitos do TMAP quando associado ao aconselhamento sexual. Os participantes apresentaram motivação quanto à realização do TMAP, pois a técnica só foi finalizada após a volta da continência.

Em afirmação com os autores Gerarts et al. (2016), o treinamento promoveu apenas moderada melhora da gravidade da $\mathrm{DE}$, porém esses indivíduos relataram estarem satisfeitos quanto à durabilidade do tratamento, pois além desses benefícios, tornou-se possível à aproximação de pessoas que vivenciaram a mesma experiência. $\mathrm{O}$ aconselhamento permitiu que esses indivíduos falassem abertamente sobre sexualidade, além de contribuir com a intimidade do casal, porque as técnicas utilizadas ajudaram nas habilidades e na estimulação íntima, aumentando o desejo e satisfação sexual.

As intervenções fisioterapêuticas não possibilitam a recuperação completa da função sexual desses pacientes, dessa forma, é de grande importância à utilização de um programa utilizando várias técnicas fisioterapêuticas associadas, pois a função sexual requer condutas mais rigorosas e específicas, sendo necessário o envolvimento de uma equipe multidisciplinar. vale lembrar que existem outros fatores envolvidos que vão contribuir para DE. Ressalta-se que, assim como a IU, a DE necessita precocemente de tratamento para resultados mais favoráveis.

\section{CONSIDERAÇÕES FINAIS}

Através dos estudos encontrados, conseguimos analisar quais as intervenções fisioterapêuticas utilizadas no pós-cirúrgico da PR, podendo destacar: o BFB, EE, terapia comportamental, pilates e TMAP; todas resultam em benefícios comuns quando iniciado o tratamento precocemente.

A melhora da função miccional foi um dos maiores benefícios entre as técnicas, porém os benefícios em relação à disfunção sexual é algo ainda em discussão, pois a maioria das intervenções apresentou apenas uma minimização da gravidade, sendo possível observar também que não há consenso quanto aos benefícios em relação à QV.

Sugere-se que sejam necessários maiores estudos envolvendo o tratamento conservador, principalmente se tratando da disfunção sexual em pacientes prostatectomizados, pois devido à idade avançada e a cirurgia afetar estruturas nervosas importantes para o retorno dessa função, a reabilitação é um processo difícil,

Nota-se a importância do envolvimento de uma equipe interdisciplinar para que se possa integrar o tratamento de forma holística, pois são pacientes que apresentam sérias complicações psicossociais, diante disso, diferentes profissionais devem estar informados para que possam prestar assistência adequada ao paciente e à família.

O atual estudo não tem a finalidade de exaurir a investigação sobre o tema, ao contrário, confia que se faz necessário aumentarmos esforços em questionamentos acerca do contexto da atuação da 
fisioterapia pélvica no tratamento das disfunções miccionais e sexuais em pacientes prostatectomizados e apresentar resultados para fortalecer o papel do profissional fisioterapeuta na prática cotidiana, promovendo uma assistência de qualidade.

\section{REFERENCIAS}

AHMED, M. T.; MOHAMMED, A. H.; AMANSOUR, A. Effect of Pelvic Floor Electrical Stimulation and Biofeedback on the Recovery of Urinary Continence after Radical Prostatectomy. Turkish Journal of Physical Medicine \& Rehabilitation/Turkiye Fiziksel Tip ve Rehabilitasyon Dergisi, v. 58, n. 3, p. 170-176, 2012.

BISPO, A. P.; NASCIMENTO, S. A.; SOLER, R. P. D. Efeitos da electroestimulação funcional na recuperação da função erectiva após prostatectomia radical bilateral com sparing nervosa: estudo randomizado controldado por SHAM. The Journal of Urology, v. 201, n. Supl. 4, p. e487-e487, 2019.

CARVALHO, M. R.; SILVA, F. A. M. N.; SILVEIRA, I. A. Alternative therapies for early recovery of post-prostatectomy urinary continence: systematic review. Enfermería Global, n. 50, p. 571, 2018.

GERAERTS, I. et al. Pelvic floor muscle training for erectile dysfunction and climacturia 1 year after nerve sparing radical prostatectomy: a randomized controlled trial. International Journal of Impotence Research, v. 28, n. 1, p. 9-13, 2016.

GOODE, P. S. et al. Behavioral therapy with or without biofeedback and pelvic floor electrical stimulation for persistent postprostatectomy incontinence: a randomized controlled trial. Jama, v. 305, n. 2, p. 151-159, 2011.

GOULART, D. M. M.; MIRANZI, M. A. S.; GOULART P. E. N. Qualidade de vida em pacientes submetidos à prostatectomia radical. Revista Eletrônica de Enfermagem, v. 16, n. 3, p. 625-34, 2014.

GOMES, C. S. et al. The effects of Pilates method on pelvic floor muscle strength in patients with post-prostatectomy urinary incontinence: A randomized clinical trial. Neurourology and Urodynamics, v. 37, n. 1, p. 346-353, 2018.

INSTITUTO NACIONAL DO CÂNCER. Saúde do Homem, 2020.

KANNAN, P. et al. Effectiveness of physiotherapy interventions for improving erectile function and climacturia in men after prostatectomy: a systematic review and meta-analysis of randomized controlled trials. Clinical Rehabilitation, v. 33, n. 8, p. 1298-1309, 2019a.

KANNAN, P. et al. Pelvic floor muscle training alone and in combination with biofeedback and/or electrical stimulation for urinary incontinence following prostatectomy: a meta-analysis. Physiotherapy, v. 105, n. Supl.1, p. e26-e27, 2019b.

KARLSEN, R. V. et al. Feasibility and acceptability of couple counselling and pelvic floor muscle training after operation for prostate cancer. Acta Oncologica, v. 56, n. 2, p. 270-277, 2017. 
KIM, J. K. et al. Efect of personalized extracorporeal biofeedback devide for pevice floor muscle training on urinary incontinence after robot-assisted radical prostatectomy: a randomized controlled trial. The Journal of Urology, v. 201, n. Supl. 4, p. e739-e739, 2019.

LAURIENZO, C. E. et al. Pelvic floor muscle training and electrical stimulation as rehabilitation after radical prostatectomy: a randomized controlled trial. Journal of Physical Therapy Science, v. 30, n. 6, p. 825-831, 2018.

MARIOTTI, G. et al. Recuperação da continência urinária após prostatectomia radical com estimulação elétrica precoce e tardia do assoalho pélvico e tratamento associado ao biofeedback. Urologia, v. 86, n. 1, p. 115-121, 2015.

MCANINCH, J. W.; LUE, T. F. Urologia geral de Smith e Tanagho. 18. ed. AMGH, 2014.

MENDES, K. D. S.; SILVEIRA, R. C. D. C. P.; GALVÃO, C. M. Revisão integrativa: método de pesquisa para a incorporação de evidências na saúde e na enfermagem. Texto \& Contexto Enfermagem, v. 17, n. 4, p. 758-764, 2008.

NILSSEN, S. R. et al. Does physiotherapist-guided pelvic floor muscle training increase the quality of life in patients after radical prostatectomy? A randomized clinical study. Scandinavian Journal of Urology and Nephrology, v. 46, n. 6, p. 397-404, 2012.

PEDRIALI, F. R. et al. Is pilates as effective as conventional pelvic floor muscle exercises in the conservative treatment of post-prostatectomy urinary incontinence? A randomised controlled trial. Neurourology and Urodynamics, v. 35, n. 5, p. 615-621, 2016.

PEREZ, F. S. B. et al. Effects of Biofeedback in the Prevention of Urinary Incontinence and Erectile Dysfunction after Radical Prostatectomy. Fronteiras em oncologia, v. 8, p. 20, 2018.

PROTA, C. et al. Early postoperative pelvic-floor biofeedback improves erectile function in men undergoing radical prostatectomy: a prospective, randomized, controlled trial. International Journal of Impotence Research, v. 24, n. 5, p. 174, 2012.

RIBEIRO, L. H. et al. Long-term effect of post-operative pelvic floor biofeedback on continence in men undergoing radical prostatectomy: a prospective, randomized, controlled study. The Journal of Urology, v. 184, n. 3, p. 1034-1039, 2010.

SANTOS, A. S. et al. Eletroestimulação na incontinência urinária pós prostatectomia radical. Fisioterapia Brasil, v. 17, n. 1, p. 50-55, 2016.

SAYILAN, A. A.; ZBAŞ, A. The Effect of Pelvic Floor Muscle Training On Incontinence Problems After Radical Prostatectomy. American Journal of Men's Health, v. 12, n. 4, p. 1007-1015, 2018.

SCARPELINI, P. et al. Protocolo de ginástica hipopressiva no tratamento da incontinência urinária pós-prostatectomia: relato de caso. Revista UNILUS Ensino e Pesquisa, v. 11, n. 23, p. 90-95, 2014.

SIGHINOLFI, M. C. et al. Potential efficacy of pelvic floor rehabilitation treatment for postradiological prostatectomy incontinence, climaturia and erectile dysfunction: a series of cases. $\mathbf{O}$ Jornal de Medicina Sexual, v. 6, n. 12, p. 3496-3499, 2009. 
SOUZA, M. T.; SILVA, M. D.; CARVALHO, R. Revisão integrativa: o que é e como fazer. Einstein, v. 8, n. 1, p. 102-106, 2010.

TANAGHO, E. A.; MCANICH, J. M. Urologia Geral de Smith e Tanagho. 10. ed. Porto Alegre: Manole, 2006. 768 p.

ZAIDAN, P.; MULLER, J. F.; SILVA, E. B. Electrical stimulation, elvic floor muscle exercises, and urinary in continence in post-prostatectomy patients: controlle drandomize double-blind experiment. International Journal of Current Research, v. 8, n. 11, p. 41859-41863, 2016.

ZAIDAN, P.; SILVA, E. B. Electrostimulation, response of the pelvic floor muscles, and urinary incontinence in elderly patients post prostatectomy. Fisioterapia e Pesquisa, v. 27, n. 1, p. 93-100, 2014. 